\title{
Factores de riesgo para enfermedades crónicas en universitarias del área de la salud
}

\author{
Health area university women non-transmis- \\ sible chronic diseases risk factors
}

Mtra. Guillermina Arenas Montaño*, Mtra. María de los Angeles Torres Lagunas **, Mtro. Magdaleno Santillán Acosta***, Dra. Alba Luz Robles Mendoza**** *Profesora de Carrera Asociada "C" de la Facultad de Estudios Superiores Iztacala de la UNAM **

Profesora de Carrera Asociada "C" de ENEO-UNAM, ***Profesor Asociado "C" de la Facultad de Estudios Superiores Iztacala de la UNAM, ${ }^{* * * *}$ Profesora de Asignatura "B" de la Facultad de Estudios Superiores Iztacala de la UNAM.

\section{Resumen}

El propósito del estudio fue identificar la presencia de factores de riesgo para enfermedades crónicas no transmisibles (ECNT) en un grupo de 37 universitarias de la Facultad de Estudios Superiores Iztacala de la UNAM, con el fin de diseñar una estrategia de intervención. Material y métodos: Es un estudio y cuantitativo, descriptivo y transversal. Resultados: Se encontró que los factores de riesgo más comunes en esta población fueron los antecedentes hereditarios de diabetes, hipertensión arterial y cáncer. Entre los factores personales que destacaron, se encuentra la inactividad física deportiva, el sobrepeso, obesidad, sedentarismo y la jornada laboral prolongada. En los Papanicolaou realizados se encontró proceso infeccioso. Discusión: El factor obesidad tuvo mayor prevalencia en los grupos de académicas y administrativas. Las académicas duermen menos horas, por dedicar más tiempo al trabajo remunerado. Se encontraron tabúes relacionados

The purpose of this study was to identify the presence of risk factors for chronic non-communicable diseases (ECNT) university women from the Iztacala Superior Studies within a group National Autonomous University of Mexico (UNAM) in order to design an intervention strategy. Materials and methods: This is a descriptive study, cross-cutting quantitatively. Results: We found that the most common risk factors in the population were the hereditary background of diabetes, hypertension and cancer. Among the factors that emphasized personal, physical inactivity is a sport, overweight, obesity, a sedentary lifestyle and long working hours. In all women who were Pap found the infectious process. Discussion: The factor of obesity was most prevalent in the academic and administrative groups. The academic sleep fewer hours because they spend more time on paid work. These women were found tabues related to the genital region, which will limit the early realization of preventive studies con la región genital, que limitan la realización de exámenes preventivos. Conclusiones: Se observó, alta prevalencia de factores de riesgo para desarrollar ECNT. Se propuso un plan de intervención para promover estilos de vida saludables, considerar a la facultad como campo de práctica comunitaria, donde se lleve a cabo el fomento para la salud y la protección específica de enfermedades, a través acciones formativas que promuevan el consumo de dietas personalizadas, educación sexual, prevención de adicciones, seguimiento de casos y el uso de las instalaciones deportivas, favoreciendo así la salud integral de las universitarias.

Palabras clave: Factores de riesgo, enfermedad crónica no transmisible, estilos de vida, autocuidado, género y salud, universitarias. for cervical cancer. Conclusions: We observed high prevalence of risk factors for developing ECNT in university women studied, which proposed a plan for promoting healthy lifestyles that include conducting educational activities, considering it to the school as a field of more practical community clinic, which is conducted for health promotion with educational activities that promote healthy diets, sex education, prevention of addictions; tracking cases, promoting the use of the sports facilities within of the Faculty. All of the previous in order to favour the integral health of academic and administrative university women of the Iztacala superior studies school of the National Autonomous University of Mexico.

Key words: Risk factors, non-transmissible chronic illness, life styles self-care, gender and health, university. 


\section{INTRODUCCIÓN}

En México, más del 50\% de la población entre 20 y 69 años de edad (> 20 millones), es portadora de por lo menos una de las ECNT ${ }^{1}$ y más de la mitad lo desconoce. Estas enfermedades constituyen un problema creciente a nivel mundial y se atribuyen a los cambios, entre los que destacan, el aumento de la expectativa de vida y la práctica de estilos de vida no saludables introducidos por la modernización y la globalización; tales como sedentarismo, dietas inadecuadas, estrés, tabaquismo, alcoholismo, exceso de trabajo físico e intelectual y asunción de roles estereotipados culturalmente entre otros.

La salud es un proceso complejo determinado por factores biológicos, psicológicos y sociológicos en el que el género, por su interrelación con todos ellos, tiene un papel importante. Rohlfs y Borrell ${ }^{2}$, han demostrado que las conductas relacionadas con la salud son diferentes en las mujeres y en los hombres, aunque son cambiantes con el tiempo. Culturalmente los hombres han desarrollado conductas de riesgo físico (violencia, abuso de sustancias adictivas, velocidad en la conducción de automóviles etc.) con mayor frecuencia que las mujeres. Estos factores de riesgo para la salud, se manifiestan en la etapa media de la vida de los hombres y los conducen a una muerte prematura.

En relación con las mujeres, son "sus" roles socioculturales aprendidos los que les suelen generar problemas de salud física y mental. Pues son condiciones que se extienden a lo largo de toda la vida, sin producir la muerte prematura, pero sí limitaciones de larga duración. Para muchas mujeres el incorporarse al trabajo remunerado no siempre es del todo gratificante, ya que implica una doble jornada laboral, por un lado el trabajo público y por otro el trabajo privado de casa. Por esta razón, muchas de ellas presentan un mayor desgaste físico y mental, que las lleva a estados de agotamiento, enfermedad y depresión ${ }^{3}$.

Chávez y Casanueva ${ }^{4}$ han observado que frecuentemente las mujeres olvidan o aplazan el cuidado de su cuerpo y su salud. Suele ser común que cuando las enfermedades se dan en un plano muy visible, es cuando ellas empiezan a preocuparse y ocuparse del cuidado de su persona. La carga exagerada de trabajo para las mujeres, representa un obstáculo para alcanzar su pleno desarrollo como seres humanos. Pedrero ${ }^{5}$ señala que en el mundo, dos de cada tres mujeres sufren de anemia crónica, desnutrición, fatiga severa y como consecuencia, presentan una mayor susceptibilidad a infecciones de los aparatos respiratorio y reproductivo.
Estadísticas del Instituto Nacional de Geografía e Informática (INEGI) en México ${ }^{6}$, muestran que la esperanza de vida se ha incrementado, las mujeres viven en promedio más años que los hombres. En 1930, la esperanza de vida para las personas de sexo femenino era de 35 años y para el masculino de 33; para el 2005 la tendencia cambió a 77 y 73 años, respectivamente. El hecho de que las mujeres tengan una mayor supervivencia no equivale a una mejor salud, por el contrario, las observaciones empíricas indican que las mujeres tienden a experimentar mayor morbilidad que los hombres a lo largo del ciclo vital. Esta morbilidad se expresa en incidencias más altas de trastornos agudos, una mayor prevalencia de enfermedades crónicas no mortales, y niveles más altos de discapacidad en el corto y largo plazo ${ }^{7}$.

Los datos de la Encuesta Nacional de Salud 2000, dan cuenta de la prevalencia de algunas enfermedades en la población femenina premenopáusica; por ejemplo, la hipertensión arterial es una enfermedad común que alcanza a una de cada cinco mujeres en el grupo de 40 a 49 años de edad. La artritis reumatoide es un problema más frecuente entre las mujeres que entre los hombres, sobre todo en las mujeres con peso excesivo.

El sobrepeso y la obesidad, son factores de riesgo ${ }^{9}$ para diversas enfermedades crónicas y degenerativas, las cuales incluyen: a la diabetes mellitus, hipertensión arterial sistémica, hiperlipidemias, enfermedades respiratorias, así como procesos tumorales de la glándula mamaria, del sistema digestivo y genitourinario, todas ellas principales causas de morbilidad en la población adulta

En relación con las adicciones al tabaco y alcohol de las mujeres, al realizar una comparación de las encuestas nacionales de adicciones de 1998 y $2002^{10}$ se observa un aumento en los índices de consumo. El Cáncer Cervicouterino es el tipo más común de neoplasias entre las mujeres de América Latina y el Caribe ${ }^{11}$. Cada año, se diagnostican cerca de 77.000 nuevos casos y unas 30.000 mujeres mueren prematuramente por esta enfermedad.

Paralelamente, al realizar un análisis del modelo de atención para la salud que domina el panorama de las instituciones prestadoras de servicios de salud en nuestro país, acertamos que es el basado en el modelo biologista; el cual se caracteriza por explicar el proceso salud-enfermedad con una visión reduccionista. Es un modelo unicausal que establece una relación lineal entre el agente patógeno y la presencia de enfermedad, lo cual apoya la práctica de servicios de salud de tipo curativo, individualista, y hospitalario que busca erradicar o controlar una enfermedad 
infecciosa o crónica o bien reparar los problemas de salud, con medidas tradicionales o con procedimientos clínicos propios de la medicina curativa y olvida los aspectos sociológicos, políticos, económicos y culturales entre otros, que podrían ser los causantes de los problemas de salud o los obstáculos para la recuperación de ésta. El modelo biologicista, repercute negativamente en una posibilidad real de contar con servicios médicos oportunos y adecuados, generando expectativas contradictorias a la realidad social de salud, por la tendencia a la especialización, super-especialización y la concentración de servicios de salud en las áreas urbanas.

Con el propósito de encauzar las prácticas de salud desde el enfoque de la medicina preventiva, hacemos las siguientes precisiones conceptuales. Orem refiere que el auto cuidado es la práctica de actividades que las personas efectúan en su propio beneficio para el mantenimiento de la vida, la salud y su seguridad ${ }^{12}$. En este sentido considera el concepto "auto" como la totalidad de un individuo, incluyendo no sólo sus necesidades biológicas, sino también las psicológicas y espirituales, y el concepto "cuidado" como la totalidad de actividades que una persona realiza para mantener la salud y desarrollarse de una forma que sea favorable para su vida. La realización del autocuidado requiere de la acción intencionada y calculada, que está condicionada por la adquisición del conocimiento y de habilidades de una persona, y se basa en la premisa que las personas saben cuándo necesitan ayuda y por lo tanto, son conscientes de las acciones específicas que necesitan realizar. Sin embargo, pueden escoger entre distintas opciones en sus conductas de autocuidado; y en algunas circunstancias, la persona puede decidir, por cualquier razón, no iniciar la conducta de auto cuidado cuando es necesaria.

Fox y Goldblatt ${ }^{13}$ han encontrado que el proceso por el cual un individuo se define como enfermo y decide actuar para la atención de sus síntomas, depende de qué tan comunes y aceptados son tales síntomas en un determinado grupo. En consecuencia, las expectativas sociales que se tienen de las mujeres, están en estrecha relación con aquello que la misma mujer percibe, interioriza y define como salud y enfermedad.

El autocuidado es también un medio efectivo para el manejo de las enfermedades. Los programas educativos pueden ayudar a un grupo de personas con problemas de salud tales como obesidad, diabetes, infecciones de vías urinarias, a sentirse más tranquilos respecto a estos, al aprender conductas que eviten la enfermedad, un daño más severo, y a saber cuándo acudir a los servicios de salud. El educar a las personas acerca del autocuidado, les estimula a tomar un papel más activo respecto de su salud. Las coloca en una posición más segura, permitiéndoles tomar decisiones informadas y confiadas acerca de su beneficio personal.

Partiendo del supuesto de que en una institución educativa del área de la salud, en la que se forman profesionales encargados de fomentar y promover la educación del cuidado de la salud de la población, éstos deberían tener pocos factores de riesgo que les ocasionaran enfermedad, debido a que aplicarían en ellos mismos los conocimientos y acciones que han aprendido para el cuidado de la salud de los demás, el objetivo que planteamos en esta investigación es identificar la presencia de factores de riesgo de ECNT en un grupo de mujeres universitarias, con el fin de planificar una estrategia de intervención.

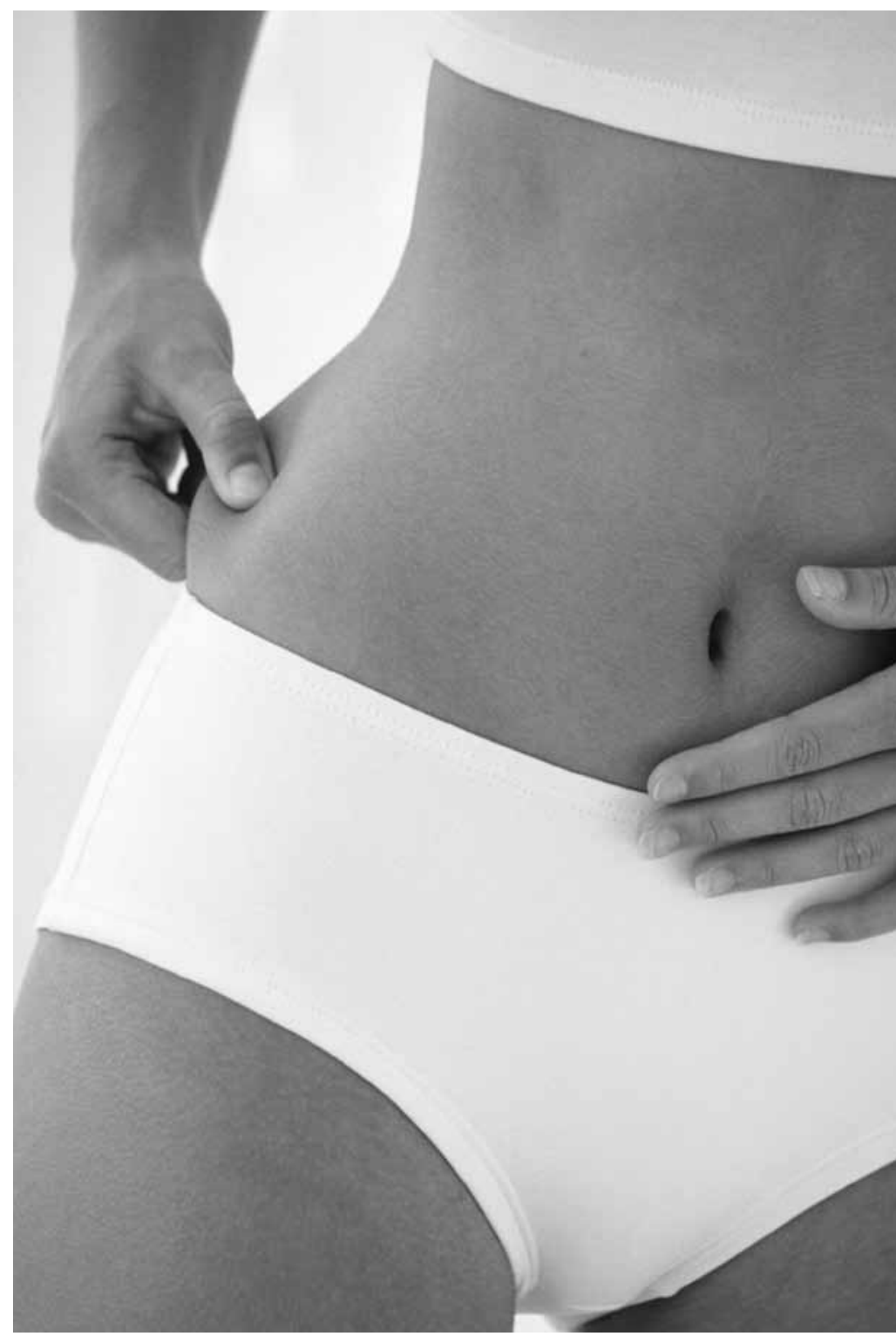




\section{MATERIAL Y MÉTODOS}

En marzo del 2006, se realizó un estudio transversal y descriptivo en la Facultad de Estudios Superiores Iztacala de la UNAM. El universo de estudio estuvo conformado por 37 mujeres adultas, todas ellas adscritas a la FESI, que acudieron por convocatoria abierta. Las mujeres fueron clasificadas en tres categorías, quedando distribuidas de la siguiente manera: 10 académicas, 12 administrativas y 15 estudiantes. El rango de edad estuvo comprendido entre los 18 y 55 años. La recolección de la información la realizaron pasantes de la Licenciatura en Enfermería, previo consentimiento informado de las participantes. Se les realizó una entrevista y fue elaborada una historia clínica, mediante la cual se identificaron factores de riesgo presentes en cada participante; estos fueron: datos de identificación, antecedentes heredo-familiares de enfermedad, antecedentes personales de enfermedad, horas dedicadas al trabajo remunerado, al sueño, y la práctica de ejercicio físico, presencia de consumo de tabaco y alcohol. Se registró talla, peso, se realizó examen general de orina (EGO) y estudio de Papanicolaou.

El sobrepeso y la obesidad se clasificaron de acuerdo con el índice de masa corporal y fue calculado conforme a los criterios de la norma Oficial mexicana para el manejo de la obesidad ${ }^{14}$. Se consideró físicamente activa a las personas que realizaban como mínimo, ejercicio durante media hora al menos tres veces por semana; o sedentarias, aquellas que no realizaban ninguna actividad física. Se diagnosticó infección de vías urinarias (IVU), cuando la orina presentaba: aspecto turbio, nitritos positivos, más de 50 leucocitos por campo y presencia de bacterias. Para el análisis estadístico se utilizó estadística descriptiva a través de frecuencias, proporciones y medidas de tendencia central.

\section{RESULTADOS}

Del grupo de académicas: el $60 \%$ se encontró en el rango de edad entre 46 a 50 años, el 90 \% tenía grado académico de Licenciatura, el estado civil que predominó fue el de casadas con el $60 \%$. Dentro de la carga hereditaria de enfermedades, se encontró la diabetes mellitus en $60 \%$, hipertensión arterial sistémica en 50\%, y cáncer en $50 \%$. (Tabla 1). En los antecedentes personales de enfermedad, predominó la hipertensión arterial, hiperlipidemia, y lumbalgias, cada una con un $20 \%$. El $10 \%$ presentó sobrepeso y un $40 \%$ presentó obesidad. El hábito de consumo de alcohol, determinado por la frecuencia de su ingestión, estuvo presente en el $30 \%$ y de tabaco el $20 \%$. El promedio de horas que ellas dedicaban para realizar trabajo remunerado fue de 9; y el promedio de horas que dedicaban para dormir fue de 6 . El $70 \%$ no practicaba ninguna actividad de acondicionamiento físico ni la práctica de algún deporte. En tres académicas que se efectuaron el Papanicolaou, se observaron alteraciones en cuello uterino: erosiones, proceso inflamatorio e infecciones vaginales. Siete académicas no aceptaron la realización del Papanicolaou, cinco por haberlo realizado un año antes con resultados normales y dos porque "no les gustaba". En sólo un EGO se detectó infección de vías urinarias.

TABLA 1.

Antecedentes heredo-familiares de enfermedades crónico degenerativas

\begin{tabular}{|l|c|c|c|}
\hline & $\begin{array}{c}\text { Diabetes } \\
\text { mellitus \% }\end{array}$ & $\begin{array}{c}\text { Hipertensión ar- } \\
\text { terial sistémica \% }\end{array}$ & $\begin{array}{c}\text { Cáncer } \\
\%\end{array}$ \\
\hline Académicas & 60 & 50 & 50 \\
\hline $\begin{array}{l}\text { Administrati- } \\
\text { vas }\end{array}$ & 60 & 60 & 30 \\
\hline Alumnas & 80 & 33 & 40 \\
\hline
\end{tabular}

En el grupo de administrativas: el $40 \%$ se encontró en el rango de edad de 46 a 50 años; su nivel académico fue de bachillerato en un $80 \%$, el estado civil que predomino fue el de casadas con el $40 \%$. Dentro de la carga hereditaria de enfermedades, se encontró la diabetes mellitus en un $60 \%$, hipertensión arterial sistémica en el $60 \%$ y cáncer en el 30\%. En los antecedentes personales de enfermedad, predominó la gastritis en el 33\%, la hipertensión arterial sistémica en el $25 \%$ y las infecciones de vías urinarias en el 16\%. El $20 \%$ presentó sobrepeso y el 40\%, obesidad. (Tabla 2). El hábito del consumo de alcohol fue del $40 \%$ y de tabaco en el $25 \%$. Los promedios de horas que dedicaban para realizar trabajo remunerado fueron de 9; y para dormir de 8 . El $75 \%$ no practicaba ninguna actividad de acondicionamiento físico ni la práctica de algún deporte. En tres personas que se efectuaron el Papanicolaou, se observó alteración en cuello uterino: procesos inflamatorios, infecciones vaginales y en una, presencia del virus del papiloma

TABLA 2.

Población con sobrepeso y obesidad

\begin{tabular}{|l|c|c|}
\hline & $\begin{array}{c}\text { Sobrepeso } \\
\%\end{array}$ & $\begin{array}{c}\text { Obesidad } \\
\%\end{array}$ \\
\hline Académicas & 10 & 40 \\
\hline Administrativas & 20 & 40 \\
\hline Alumnas & 20 & 13 \\
\hline
\end{tabular}


humano (VPH). Siete administrativas no aceptaron la realización del Papanicolaou, cuatro por "haberlo realizado un año antes con resultados normales", dos porque "no les gustaba" y una porque "le daba miedo". En sólo un EGO, se detectó infección de vías urinarias.

Respecto al grupo de alumnas, el $40 \%$ se encontró en el rango de edad de 21 a 25 años; su nivel académico fue de estudiantes de la Licenciatura en el $80 \%$. El estado civil que predomino fue el de solteras con el $80 \%$. Dentro de la carga hereditaria de enfermedades, se encontró: la diabetes mellitus en el $80 \%$, cáncer con $40 \%$ e hipertensión arterial sistémica en $33 \%$. En los antecedentes personales de enfermedad, predominó la migraña en un $20 \%$, gastritis con $13 \%$ y quiste de ovario en $13 \%$. El hábito de consumo de alcohol fue de $26 \%$ y de tabaco de $13 \%$. El $20 \%$ presentó sobrepeso, 13\% tuvo obesidad. El promedio de horas que dedicaban para realizar trabajo remunerado fue de 4 , y el que dedicaban para dormir, de 7 . El $87 \%$ no practicaba ninguna actividad de acondicionamiento físico ni la práctica de algún deporte. (Tabla 3). En las cinco personas que se efectuaron el Papanicolaou, se observó alteración en cueIlo uterino: procesos inflamatorios e infecciones vaginales. En una se observó la presencia del (VPH). Siete alumnas no aceptaron la realización del Papanicolaou, cuatro porque "nunca habían tenido relaciones sexuales" dos 2 "sin razón" y una porque "le daba miedo". En sólo un EGO, se detectó infección de vías urinarias.

\section{TABLA 3.}

Población que dedica tiempo

para realizar actividades físicas deportivas

\begin{tabular}{|l|c|c|}
\hline & $\begin{array}{c}\text { Con actividad física } \\
\%\end{array}$ & $\begin{array}{c}\text { Sin actividad física } \\
\%\end{array}$ \\
\hline Académicas & 30 & 70 \\
\hline Administrativas & 25 & 75 \\
\hline Alumnas & 13 & 87 \\
\hline
\end{tabular}

\section{DISCUSIÓN}

La edad de las académicas y administrativas osciló entre la $4^{\mathrm{a}}$. Y $5^{\mathrm{a}}$. década de su vida, mientras que la de las estudiantes fue de entre 21 y 25 años. Sin embargo, las estudiantes presentaron factores de riesgo similares a las primeras. En los tres grupos de mujeres encontramos factores de riesgo hereditarios para desarrollar ECNT, predominando la diabetes mellitus, la hipertensión arterial y diversos tipos de cáncer. La enfermedad que prevaleció en las académicas y las administrativas fue la hipertensión arterial y en las estudian- tes la migraña. El consumo de alcohol fue mayor que el de tabaco en los tres grupos, teniendo mayor prevalencia el de las administrativas. Tanto académicas como administrativas dedicaban 9 horas en promedio para realizar trabajo remunerado; sin embargo, las académicas dormían menos horas; en comparación con las estudiantes, que trabajaban 4 y dormían 7 horas. En relación a esto, Chávez y Casanueva mencionan que frecuentemente las mujeres olvidan o aplazan el cuidado de su cuerpo y su salud, que la carga exagerada de trabajo para ellas representa un obstáculo para alcanzar su pleno desarrollo como seres humanos. En los tres grupos, se observó una prevalencia alta de inactividad física deportiva, pero predominó en el las estudiantes. En todas las mujeres que se realizaron Papanicolaou, se encontraron procesos inflamatorios e infecciosos y en 1 administrativa y 1 estudiante, se encontró virus del papiloma humano (VPH). En mujeres de las tres categorías, hubo quienes no permitieron la realización de papanicolaou, debido a ciertos tabúes relacionados con la región genital.

Los datos derivados del presente estudio muestran una elevada prevalencia de obesidad, tomando la referencia de Gonzáles y Villalpando, ésta es un factor de riesgo para diversas enfermedades crónicas y degenerativas, las cuales incluyen: a la diabetes mellitus, hipertensión arterial, hiperlipidemias, así como procesos tumorales de la glándula mamaria, del sistema digestivo y genitourinario, En congruencia con esta información, los grupos de mujeres que estudiamos presentan riesgo mayor para desarrollar este tipo de enfermedades.

\section{CONCLUSIONES}

Se pudo observar que los grupos de mujeres estudiadas, presentaron alta prevalencia de factores de riesgo para desarrollar ECNT. Los estilos de vida no saludables en esta población, se ven reflejados en la obesidad, que es un problema grave en las académicas y administrativas; en general su actividad fue sedentaria. El consumo de alcohol es una práctica dañina severa, aún considerando que este factor de riesgo actuará en períodos prolongados. Suponiendo que estos grupos de mujeres se someten a un alto grado de estrés laboral en cuanto a las horas de trabajo, principalmente el grupo de académicas, por las horas extras de trabajo que requiere su labor docente desarrollada en el espacio del hogar, el riesgo de desarrollar ECNT es muy alto, si no se toman las medidas necesarias preventivas. Se propone realizar actividades educativas para promover estilos de vida saludables que involucren a los 
tres grupos de mujeres. Efectuar seguimiento y control de aquellas personas de mayor riesgo, a través de programas multidisciplinarios de prevención y control de enfermedades crónicas no transmisibles, realizados dentro de la institución educativa por los propios alumnos y profesores. Tomar en consideración a la institución educativa como un campo más de práctica clínica comunitaria, donde se realicen las acciones del primer nivel de prevención como son: educación en el consumo de dietas más saludables, conocimiento de su sexualidad, conocimiento de las adicciones, control de peso, fomento de la actividad física en la vida cotidiana y uso de las instalación deportivas dentro de la Facultad, asistencia a la clínica antitabaco y consejería, entre otras. Todo esto con el, propósito de favorecer la salud integral de las mujeres universitarias.

"Agradecimiento por el apoyo recibido de PAPCA 2006 de la FESI“

\section{REFERENCIAS BIBLIOGRÁFICAS}

1 Velásquez, M. Rosas, P. Lara, E. Pastelón, Sánchez, C. Tapia, C. "Prevalencia e interrelación de enfermedades crónicas no transmisibles y factores de riesgo cardiovascular en México" Resultados finales de la Encuesta Nacional de Salud (ENSA) 2000. en Arch. Cardiol. Mex. 2003; 73 (1): 6277

2 Rohlfs, C. Borrell, C. "La importancia de la perspectiva de género en las encuestas de salud"en: Gaceta Sanitaria. Barcelona, 2000; 14 (2): 146-155.

3 Noriega, M. Gutiérrez, G. Méndez, I. Pulido, M."Las trabajadoras de la salud: vida, trabajo y trastornos mentales". Cuadernos de Saúde Pública Rio de Janeiro 2004; 20 (5): 1361-1372

4 Chávez, M M. Casanueva, "El Uso de servicios preventivos de salud materno-infantil en un grupo de mujeres mexicanas" en Rev. Salud Pública Bogota, 2005; 7 (1): 16-25.

5 Pedrero M. Cinco dimensiones sobre la situación de la mujer mexicana: legal, política, bienestar, trabajo y fecundidad. Aportes de investigación. Cuernavaca: Universidad Nacional Autónoma de México/Centro Regional de Investigaciones Multidisciplinarias. 1992; 217-222.

6 INEGl.- "Estadísticas a propósito del Día internacional de la Mujer. 2006 INEGI. Estadísticas sociodemográficas. Dinámica de la población"

7 Verbrugge LM. Pathways in health and death. En: Apple R, ed. Women, health and medicine in America. New York: Garland Publishing; 1990.
8.- Velásquez, M. Rosas, P. Lara E. Hipertensión arterial en México: Resultados de la Encuesta Nacional de Salud 2000. en Arch. Cardiol. Mex. 2002; 72 (1): 71-84.

9.- Gonzáles- Villalpando C, Stern PM. La obesidad como riesgo cardiovascular en México. en Rev Investigación Clínica 1993; 45 (1): 13-21.

10.- Sistema de Vigilancia Epidemiológica de las Adicciones SISVEA Informe del Sistema de Vigilancia Epidemiológica de las Adicciones SISVEA México 2002; 19-23.

11.- Análisis de la situación del cáncer cérvico-uterino en América Latina y el Caribe. Ed. Organización Panamericana de la Salud. Unidad de Enfermedades no transmisibles, Washington, D.C; 2006; 2-10.

12.- Orem, D. E. Modelo de Orem: Conceptos de Enfermería en la práctica. Pennsylvania, Masson y Salvat; 1993; 419-421.

13.- Fox, A. Goldblatt. O, Social class mortality differentials: Artefact, selection or life circumstances. J Epidemiol Community Health 1985; 39 (1):1-8

14.-Secretaría de Salud. Norma oficial mexicana NOM-174SSA1-1998, para el manejo integral de la obesidad. Rev Med .IMSS 2000; 38 (5): 397-403.

\section{DIRECCIÓN PARA CORRESPONDENCIA}

Mtra. Guillermina Arenas Montaño: guillerenas@yahoo.com.mx

Mtra. María de los Angeles Torres L.: angelestorres2007@gmail. com

Mtro. Magdaleno Santillán A.: msant@campus.iztacala.unam.mx Dra. Alba Luz Robles M.: albpsic@campus.iztacala.unam.mx

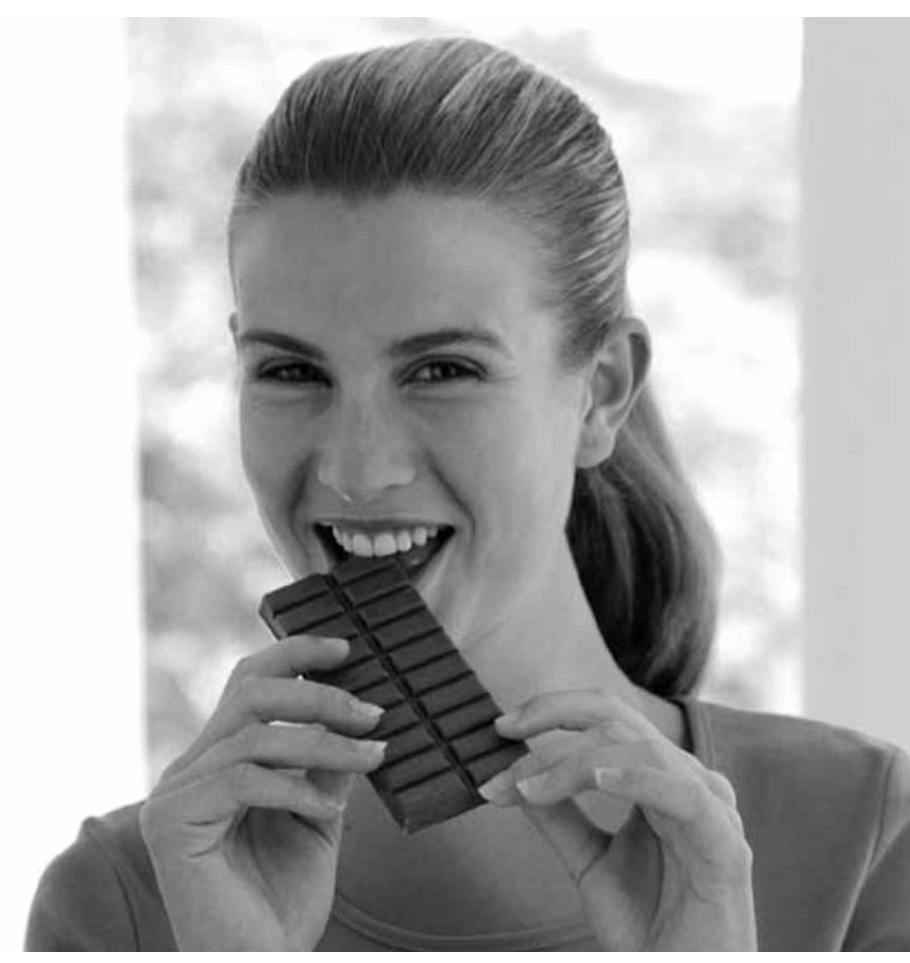

